\title{
Analytical expressions for field astigmatism in decentered two mirror telescopes and application to the collimation of the ESO VLT
}

\author{
L. Noethe ${ }^{1}$ and S. Guisard ${ }^{2}$ \\ 1 European Southern Observatory, Karl-Schwarzschild-Str. 2, Garching, Germany \\ e-mail: lnoethe@eso.org \\ 2 European Southern Observatory, Casilla 19001, Santiago 19, Chile \\ e-mail: sguisard@eso.org
}

Received January 17; accepted March 9, 2000

\begin{abstract}
We derive formulae for all parameters defining the field astigmatism of misaligned two mirror telescopes with arbitrary geometries and with stop positions anywhere on the line connecting the vertices of the two mirrors. The formulae show explicitly the dependence of the field astigmatism on the fundamental design parameters and characteristics of the telescope and on the stop position. Special attention is given to the particular case where such a schiefspiegler has been corrected for coma at the field center. In addition, we study the effects of the practical definition that the center of the field is the center of the adapter. Following a recent paper by McLeod, where the field dependence of astigmatism is used to collimate a Ritchey-Chretien telescope with the stop at the primary mirror, we apply our formulae to the Cassegrain focus of the ESO Very Large Telescope (VLT), where the stop is at the secondary mirror and the telescope is only corrected for spherical aberration. We present measurements of the field astigmatism and discuss the accuracy of the collimation method.
\end{abstract}

Key words: telescopes

\section{Introduction}

A general theory of low order field aberrations of decentered optical systems has been given by Shack \& Thompson (1980). In particular it has been shown that the general field dependence of third order astigmatism can be described by a binodal pattern, known as ovals of Cassini. Only for special cases such as a centered system do the two nodes coincide and the field dependence reduces to the well known rotationally symmetric pattern with a quadratic dependence on the distance to the field center. These general geometric properties have been used by McLeod (1996), starting from equations by Schroeder (1987), for the alignment of an aplanatic two mirror telescope. We shall recall this method briefly.

In a Cassegrain telescope the absence of decentering coma in the center of the field does not imply that the optical axes of the primary (M1) and the secondary mirrors (M2) coincide. The axes of the primary and the secondary mirrors must intersect at the coma free point, but the axis of M2 may still form an angle $\alpha$ with respect to the axis of M1. For this case McLeod showed that the components $Z_{4}$ and $Z_{5}$ of third order astigmatism of a two mirror telescope with the stop at the primary mirror for a field angle $\theta$ with components $\theta_{x}$ and $\theta_{y}$ are given by

$$
\begin{aligned}
Z_{4}^{\mathrm{sys}}= & B_{0}\left(\theta_{x}^{2}-\theta_{y}^{2}\right)+B_{1}\left(\theta_{x} \alpha_{x}-\theta_{y} \alpha_{y}\right) \\
& +B_{2}\left(\alpha_{x}^{2}-\alpha_{y}^{2}\right) \\
Z_{5}^{\mathrm{sys}}= & 2 B_{0} \theta_{x} \theta_{y}+B_{1}\left(\theta_{x} \alpha_{y}+\theta_{y} \alpha_{x}\right)+2 B_{2} \alpha_{x} \alpha_{y} .
\end{aligned}
$$

$B_{0}$ is the coefficient of field astigmatism for a centered telescope, $B_{1}$ and $B_{2}$ only appear in decentered systems. Numerical values for $B_{0}, B_{1}$ and $B_{2}$ were obtained by using general formulae for field astigmatism of individual mirrors and adding the effects of the two mirrors. The values for $\alpha_{x}$ and $\alpha_{y}$ could then be obtained from measurements of $Z_{4}^{\text {sys }}$ and $Z_{5}^{\text {sys }}$ in the field of the telescope.

For a centered two mirror telescope Wilson (1996) has derived expressions for the low order field aberrations including third order astigmatism $\left(B_{0}\right)$ showing the dependence on fundamental design parameters and optical properties of the total telescope and on the position of the stop along the optical axis. This gives more physical insight into the characteristics of field aberrations of two mirror telescopes. Similarly we derive, for decentered two mirror telescopes, explicit expressions for third order astigmatism, i.e. for the parameters $B_{1}$ and $B_{2}$, and 
discuss the field dependence of astigmatism for fundamental types of two mirror telescopes.

In a centered optical system the field center, projected towards the sky, is the direction parallel to the optical axis of M1. The formulae for the parameters $B_{0}, B_{1}$ and $B_{2}$ are initially derived for this reference system. However, in a decentered system the image of an object in the field center is not in the mechanical center of the adapter, where the instruments are located. For all measurements in the telescope, the practical field center is the center of the adapter. The evaluation of the measurements of field astigmatism has to take this difference of the origins of the reference systems into account. The structure of the Eqs. (1) and (2) will remain the same, but the parameters $B_{0}, B_{1}$ and $B_{2}$ will change and $\theta$ will denote the field angle with respect to the center of the adapter.

One case of practical interest is the collimation at the Cassegrain focus of the VLT where the stop is at the secondary mirror and the telescope is only corrected for spherical aberration. We apply our formulae to measurements of astigmatism in the field of the Cassegrain focus to determine the misalignment angles $\alpha_{x}$ and $\alpha_{y}$. In addition, the possibility to change the misalignment angles by well defined values by rotating the primary mirror around its vertex allows a measurement of the accuracy of this collimation method.

\section{Astigmatism in a misaligned telescope with an arbitrary pupil position}

\subsection{General formulation}

The general case which we study is shown in Fig. 1. The notation as well as the sign conventions for the angles and the distances are taken from Wilson (1996). The following list defines the parameters. $i=1$ stands for the primary mirror and $i=2$ for the secondary mirror.

$$
\begin{aligned}
& y_{i}: \text { semi-diameter of the mirror } i \\
& f_{i}^{\prime} \quad \text { : focal length of the mirror } i \\
& s_{\mathrm{pr} i}: \text { distance from the surface to the entrance } \\
& \text { pupil of mirror } i \\
& b_{\mathrm{s} i} \quad: \text { aspheric constant of the mirror } i \\
& n_{i}^{\prime} \quad \text { : index of the exit medium of mirror } i \\
& u_{\mathrm{pr} i} \text { : angle of the incoming principle ray with } \\
& \text { the axis of the mirror } i \\
& h_{i} \quad \text { : distance from the axis of the mirror } i \text { to } \\
& \text { the center of its entrance pupil } \\
& z \quad \text { : distance of the coma-free point from the } \\
& \text { surface of M2. }
\end{aligned}
$$

In this whole section the field center is defined as the optical axis of M1, that is $u_{\mathrm{pr} 1}=0$. The stop is located between the two mirrors at a distance $s_{\mathrm{pr} 2}$ from M2 and decentered laterally by a value $h$ from the M1 axis and $h_{2}$ from the M2 axis. The entrance pupil is located at a distance $s_{\text {pr1 }}$ behind M1 and decentered by $h_{1}$ from the
M1 axis. The vertex of the secondary mirror is decentered by a distance $\delta$ from the M1 axis and tilted by an angle $\alpha$ with respect to the M1 axis.

Initially we will only make the assumption that the lateral decenter $\delta$ and the rotation of M2 around its vertex by $\alpha$ are in the same plane.

After a bit of geometry following Fig. 1 we find:

$$
\begin{aligned}
u_{\mathrm{pr} 2} & =\left(\frac{s_{\mathrm{pr} 1}}{f_{1}^{\prime}}-1\right) u_{\mathrm{pr} 1}-\alpha-\frac{h_{1}}{f_{1}^{\prime}} \\
h_{1} & =-h \frac{s_{\mathrm{pr} 1}}{d_{1}+s_{\mathrm{pr} 2}} \\
h_{2} & =h-\delta-s_{\mathrm{pr} 2} \alpha .
\end{aligned}
$$

Knowing the distance $s_{\mathrm{pr} 2}$ from M2 to the stop in a plane between M1 and M2 one can calculate $s_{\mathrm{pr} 1}$ by

$s_{\mathrm{pr} 1}=\frac{s_{\mathrm{pr} 2}+d_{1}}{s_{\mathrm{pr} 2}+d_{1}-f_{1}^{\prime}} f_{1}^{\prime}$.

Inversely, $s_{\mathrm{pr} 2}$ is given by

$s_{\mathrm{pr} 2}=\frac{s_{\mathrm{pr} 1} f_{1}^{\prime}}{s_{\mathrm{pr} 1}-f_{1}^{\prime}}-d_{1}$.

The coefficient of the astigmatic wavefront aberration of a two mirror telescope is then given by (Schroeder 1987, page 79$)$

$\begin{aligned} c_{\mathrm{ast}} & =D_{1} y_{1}^{2}+D_{2} y_{2}^{2} \\ D_{i} & =\frac{n_{i}^{\prime}}{16 f_{i}^{\prime 3}}\left(A_{0, i} u_{\mathrm{pr} i}^{2}+A_{1, i} u_{\mathrm{pr} i} h_{i}+A_{2, i} h_{i}^{2}\right)\end{aligned}$

with the expressions for $A_{0, i}$ given by Schroeder (1987) in the Tables 5.6 and 5.9 (but adding the missing factor $1 / 2$ in the expressions for astigmatism)

$$
\begin{aligned}
& A_{0, i}=b_{\mathrm{s} i} s_{\mathrm{pr} i}^{2}+\left(2 f_{i}^{\prime}-s_{\mathrm{pr} i}\right)^{2} \\
& A_{1, i}=4 f_{i}^{\prime}-2\left(1+b_{\mathrm{s} i}\right) s_{\mathrm{pr} i} \\
& A_{2, i}=1+b_{\mathrm{s} i} .
\end{aligned}
$$

Introducing the expressions for $A_{0, i}, A_{1, i}$ and $A_{2, i}$ into Eq. (9) one gets

$$
\begin{aligned}
D_{i}= & \frac{n_{i}^{\prime}}{16 f_{i}^{\prime 3}}\left\{\left[b_{\mathrm{s} i} s_{\mathrm{pr} i}^{2}+\left(2 f_{i}^{\prime}-s_{\mathrm{pr} i}\right)^{2}\right] u_{\mathrm{pr} i}^{2}\right. \\
& \left.+\left[4 f_{i}^{\prime}-2\left(1+b_{\mathrm{s} i}\right) s_{\mathrm{pr} i}\right] u_{\mathrm{pr} i} h_{i}+\left(1+b_{\mathrm{s} i}\right) h_{i}^{2}\right\} .
\end{aligned}
$$

Our definition of the wavefront error differs by a factor -1 from the one given by Schroeder (1987), since we use the convention that a wavefront error is positive if the actual wavefront is in advance of the reference wavefront.

\subsection{Explicit expressions for astigmatism in a schiefspiegler of general form}

\subsubsection{Telescope in focal form}

In a two mirror telescope as shown in Fig. 1 the horizontal position of the stop may be anywhere to the left of the primary mirror. For the vertical position $h$ of the stop we make the simplifying assumption that the center of the 


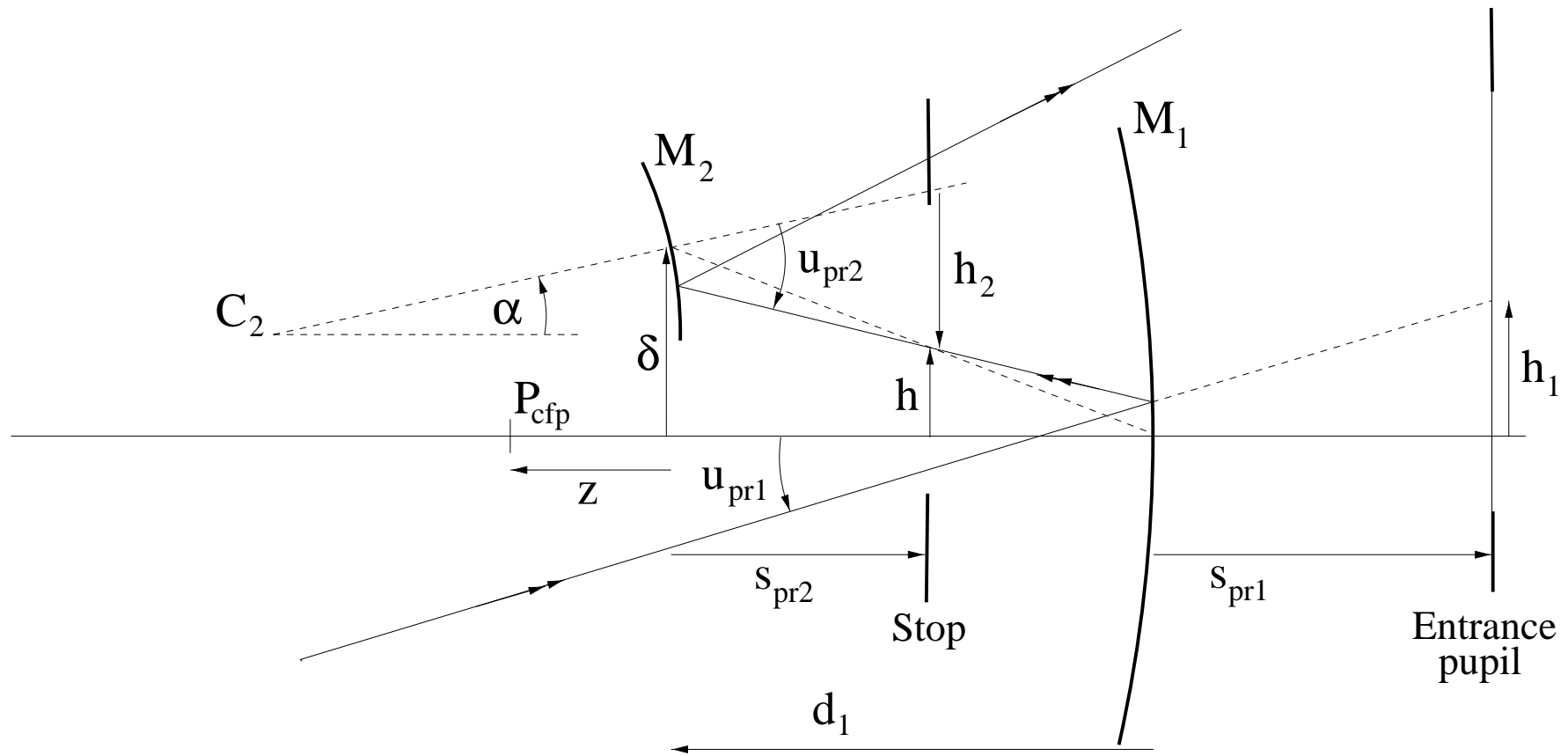

Fig. 1. Two mirror telescope with general position of M2 and general position of the stop

stop lies on the line connecting the vertices of the two mirrors. $h$ is then given by

$$
\begin{aligned}
h & =\frac{\delta}{d_{1}}\left(d_{1}+s_{\mathrm{pr} 2}\right) \\
& =\frac{\delta}{d_{1}} \frac{f_{1}^{\prime} s_{\mathrm{pr} 1}}{s_{\mathrm{pr} 1}-f_{1}^{\prime}} .
\end{aligned}
$$

With this expression for $h$ one gets for the Eqs. (3), (4) and (5)

$$
\begin{aligned}
u_{\mathrm{pr} 2} & =\frac{s_{\mathrm{pr} 1}-f_{1}^{\prime}}{f_{1}^{\prime}} u_{\mathrm{pr} 1}-\alpha+\frac{\delta}{d_{1}} \frac{s_{\mathrm{pr} 1}}{f_{1}^{\prime}} \\
h_{1} & =-\frac{\delta}{d_{1}} s_{\mathrm{pr} 1} \\
h_{2} & =\left(\frac{\delta}{d_{1}}-\alpha\right)\left(\frac{s_{\mathrm{pr} 1} f_{1}^{\prime}}{s_{\mathrm{pr} 1}-f_{1}^{\prime}}-d_{1}\right) .
\end{aligned}
$$

Introducing these expressions into Eqs. (13) and (8) and using the relationships given by Wilson (1996, Sect. 2.2.5.2)

$$
\begin{aligned}
m_{2} & =\frac{f_{2}^{\prime}}{f_{1}^{\prime}-f_{2}^{\prime}-d_{1}} \\
f^{\prime} & =m_{2} f_{1}^{\prime} \\
f_{2}^{\prime} & =\frac{L}{m_{2}+1} \\
L & =m_{2}\left(f_{1}^{\prime}-d_{1}\right) \\
y_{2} & =\frac{L}{f^{\prime}} y_{1}
\end{aligned}
$$

where $m_{2}$ is the magnification of the secondary mirror, $f^{\prime}$ the focal length of the two mirror telescope and $L$ is the distance from the secondary mirror to the focus of the telescope, one gets with $n_{1}^{\prime}=-1$ and $n_{2}^{\prime}=1$ for the coefficients of the astigmatic wavefront aberration

$$
\begin{aligned}
c_{\mathrm{ast}}= & C_{0} u_{\mathrm{pr} 1}^{2}+C_{1} u_{\mathrm{pr} 1}+C_{2} \\
C_{0}= & \frac{1}{4}\left(\frac{y_{1}}{f^{\prime}}\right)^{2}\left\{\frac{f^{\prime}}{L}\left(f^{\prime}+d_{1}\right)\right. \\
& +\frac{d_{1}^{2}}{L} \xi+\frac{s_{\mathrm{pr} 1}}{f^{\prime}}\left(f^{\prime}+2 d_{1} \xi\right) \\
& \left.+\left(\frac{s_{\mathrm{pr} 1}}{f^{\prime}}\right)^{2}\left(-f^{\prime} \zeta+L \xi\right)\right\} \\
C_{1}= & \frac{1}{4}\left(\frac{y_{1}}{f^{\prime}}\right)^{2} \\
& \left\{\alpha\left[\left(m_{2}+1\right)\left(f+L+d_{1}\right)-\frac{s_{\mathrm{pr} 1}}{f^{\prime}} L\left(m_{2}^{2}-1\right)\right]\right. \\
& +\delta\left[\left(m_{2}+1\right)^{2}\left(1+\frac{d_{1}}{2 L}\left(m_{2}+1\right)\left(1+b_{\mathrm{s} 2}\right)\right)\right. \\
& +\frac{s_{\mathrm{pr} 1}}{f^{\prime}} \frac{2}{d_{1}}\left(d_{1} \xi+\frac{f^{\prime}}{2}\right. \\
& \left.-\frac{d_{1}}{4}\left(m_{2}+1\right)^{2}\left[m_{2}-1-\left(m_{2}+1\right) b_{\mathrm{s} 2}\right]\right) \\
& \left.\left.+\left(\frac{s_{\mathrm{pr} 1}}{f^{\prime}}\right)^{2} \frac{2}{d_{1}}\left(-f^{\prime} \zeta+L \xi\right)\right]\right\} \\
C_{2}= & \frac{1}{4}\left(\frac{y_{1}}{f^{\prime}}\right)^{2} \\
& \left\{\alpha^{2} L\left(m_{2}+1\right)\right. \\
& +\alpha \delta\left[\left(m_{2}+1\right)^{2}-\frac{s_{\mathrm{pr} 1}}{f^{\prime}} \frac{L}{d_{1}}\left(m_{2}^{2}-1\right)\right] \\
&
\end{aligned}
$$


where

$$
\begin{aligned}
& +\delta^{2}\left[\frac{1}{4 L}\left(m_{2}+1\right)^{3}\left(1+b_{\mathrm{s} 2}\right)\right. \\
& -\frac{s_{\mathrm{pr} 1}}{f^{\prime}} \frac{1}{2 d_{1}}\left(m_{2}+1\right)^{2}\left[m_{2}-1-\left(m_{2}+1\right) b_{\mathrm{s} 2}\right] \\
& \left.\left.+\left(\frac{s_{\mathrm{pr} 1}}{f^{\prime}}\right)^{2} \frac{1}{d_{1}^{2}}\left(-f^{\prime} \zeta+L \xi\right)\right]\right\}
\end{aligned}
$$

$\zeta=\frac{m_{2}^{3}}{4}\left(1+b_{\mathrm{s} 1}\right)$

$\xi=\frac{\left(m_{2}+1\right)^{3}}{4}\left[\left(\frac{m_{2}-1}{m_{2}+1}\right)^{2}+b_{\mathrm{s} 2}\right]$.

The expression for $C_{0}$ is, apart from a factor needed for the conversion to Seidel coefficients, identical to the one given by Wilson (1996, Sect. 3.2.4.2).

If the stop is at the primary mirror, the parameters $C_{0}$, $C_{1}$ and $C_{2}$ should not depend on the asphericity $b_{\mathrm{s} 1}$ of the primary mirror. This can be seen from the Eqs. (24), (25) and (26), which then depend only on the asphericity $b_{\mathrm{s} 2}$ of the secondary mirror. Similarly, if the stop is at the secondary mirror, the parameters $C_{0}, C_{1}$ and $C_{2}$ should not depend on the asphericity of the secondary mirror. This can easily be verified by introducing $s_{\mathrm{pr} 2}=0$ or, equivalently, from Eq. (6), $s_{\mathrm{pr} 1} / f^{\prime}=-d_{1} / L$ into the Eqs. (24), (25) and (26).

These expressions can be considerably simplified by using corresponding expressions for other aberration coefficients of two mirror telescopes (Wilson 1996, Sects. 3.2.4.2 and 3.7.2): $c_{\text {spher }}$ of spherical aberration, $c_{\text {coma,cen }}$ of third order field coma of a centered system, and the coefficients of field independent third order coma generated by a lateral decenter by $\delta\left(c_{\mathrm{coma}, \delta}\right)$ and by a pure rotation of M2 around its vertex by $\alpha\left(c_{\text {coma }, \alpha}\right)$.

$$
\begin{aligned}
c_{\mathrm{spher}}= & \frac{1}{8}\left(\frac{y_{1}}{f^{\prime}}\right)^{4}\left(-f^{\prime} \zeta+L \xi\right) \\
c_{\mathrm{coma}, \mathrm{cen}}= & \frac{1}{2}\left(\frac{y_{1}}{f^{\prime}}\right)^{3}\left[-d_{1} \xi-\frac{f^{\prime}}{2}\right. \\
& \left.-\frac{s_{\mathrm{pr} 1}}{f^{\prime}}\left(-f^{\prime} \zeta+L \xi\right)\right] u_{\mathrm{pr} 1} \\
c_{\mathrm{coma}, \delta}= & \frac{1}{4}\left(\frac{y_{1}}{f^{\prime}}\right)^{3} \\
& {\left[\frac{1}{2}\left(m_{2}+1\right)^{2}\left[m_{2}-1-\left(m_{2}+1\right) b_{\mathrm{s} 2}\right]\right.} \\
& \left.+\frac{s_{\mathrm{pr} 1}}{f^{\prime}} \frac{2}{d_{1}}\left(-f^{\prime} \zeta+L \xi\right)\right] \delta \\
c_{\mathrm{coma}, \alpha}= & \frac{1}{4}\left(\frac{y_{1}}{f^{\prime}}\right)^{3} L\left(m_{2}^{2}-1\right) \alpha .
\end{aligned}
$$

With the further definitions

$$
\begin{aligned}
& c_{\text {coma,cen }}=c_{\text {coma,cen }}^{*} u_{\text {pr1 }} \\
& C_{0,0}=\frac{1}{4}\left(\frac{y_{1}}{f^{\prime}}\right)^{2}\left[\frac{f^{\prime}}{L}\left(f^{\prime}+d_{1}\right)+\frac{d_{1}^{2}}{L} \xi\right]
\end{aligned}
$$

$$
\begin{aligned}
C_{1,0}= & \frac{1}{4}\left(\frac{y_{1}}{f^{\prime}}\right)^{2}\left[\alpha\left(m_{2}+1\right)\left(f+L+d_{1}\right)\right. \\
& \left.+\delta\left(m_{2}+1\right)^{2}\left(1+\frac{d_{1}}{2 L}\left(m_{2}+1\right)\left(1+b_{\mathrm{s} 2}\right)\right)\right] \\
C_{2,0}= & \frac{1}{4}\left(\frac{y_{1}}{f^{\prime}}\right)^{2}\left[\alpha^{2} L\left(m_{2}+1\right)\right. \\
& \left.+\alpha \delta\left(m_{2}+1\right)^{2}+\delta^{2} \frac{1}{4 L}\left(m_{2}+1\right)^{3}\left(1+b_{\mathrm{s} 2}\right)\right] .
\end{aligned}
$$

Equations (24), (25) and (26) can be written as

$$
\begin{aligned}
C_{0}= & C_{0,0}-\frac{s_{\mathrm{pr} 1}}{y_{1}} c_{\mathrm{coma}, \mathrm{cen}}^{*}-\left(\frac{s_{\mathrm{pr} 1}}{y_{1}}\right)^{2} 2 c_{\mathrm{spher}} \\
C_{1}= & C_{1,0} \\
& -\frac{s_{\mathrm{pr} 1}}{y_{1}}\left(\frac{\delta}{d_{1}} c_{\mathrm{coma}, \mathrm{cen}}^{*}+c_{\mathrm{coma}, \alpha}+c_{\mathrm{coma}, \delta}\right) \\
& +\left(\frac{s_{\mathrm{pr} 1}}{y_{1}}\right)^{2} 4 \frac{\delta}{d_{1}} c_{\mathrm{spher}} \\
C_{2}= & C_{2,0}-\frac{s_{\mathrm{pr} 1}}{y_{1}} \frac{\delta}{d_{1}}\left(c_{\mathrm{coma}, \alpha}+c_{\mathrm{coma}, \delta}\right) \\
& +\left(\frac{s_{\mathrm{pr} 1}}{y_{1}}\right)^{2} 6\left(\frac{\delta}{d_{1}}\right)^{2} c_{\mathrm{spher}} .
\end{aligned}
$$

These equations show a nice symmetry of the stop-shift terms. The linear terms are proportional to coefficients of coma and the quadratic terms are proportional to the coefficient of spherical aberration. The total coma in the linear coefficient of $C_{1}$ is the sum of the coma for a centered system for a principal ray with the angle $\delta / d_{1}$, which, in the decentered system, is the angle of the principal ray connecting the vertices of the two mirrors, and the coma contributions from a pure decenter of M2 by $\delta$ and a pure rotation of M2 around its vertex by $\alpha$. The total coma in the linear coefficient of $C_{2}$ contains only the two latter contributions.

\subsubsection{Telescope in afocal form}

Equations (29) to (39) can be converted into equations valid for afocal telescopes, where both the total focal length $f^{\prime}$ and the position of the focus, which is linked to $L$, go to infinity. One can eliminate $f^{\prime}$ and $L$ in favour of $f_{1}^{\prime}, d_{1}$ and $m_{2}$ with the Eqs. (19) and (21) and then let $m_{2}$ go to infinity. This gives

$$
\begin{aligned}
c_{\text {spher }, \mathrm{af}}= & \frac{1}{32}\left(\frac{y_{1}}{f_{1}^{\prime}}\right)^{4}\left[-f_{1}^{\prime}\left(1+b_{\mathrm{s} 1}\right)\right. \\
& \left.+\left(f_{1}^{\prime}-d_{1}\right)\left(1+b_{\mathrm{s} 2}\right)\right] \\
c_{\text {coma }, \text { cen }, \text { af }}= & -\left[\frac{1}{8}\left(\frac{y_{1}}{f_{1}^{\prime}}\right)^{3} d_{1}\left(1+b_{\mathrm{s} 2}\right)\right. \\
& \left.+4 \frac{s_{\mathrm{pr} 1}}{y_{1}} c_{\text {spher }, \mathrm{af}}\right] u_{\mathrm{pr} 1}
\end{aligned}
$$




$$
\begin{aligned}
c_{\mathrm{coma}, \delta, \mathrm{af}}= & {\left[\frac{1}{8}\left(\frac{y_{1}}{f_{1}^{\prime}}\right)^{3}\left(1-b_{\mathrm{s} 2}\right)\right.} \\
& \left.+\frac{s_{\mathrm{pr} 1}}{y_{1}} \frac{4}{d_{1}} c_{\text {spher }, \mathrm{af}}\right] \delta \\
c_{\mathrm{coma}, \alpha, \mathrm{af}}= & \frac{1}{4}\left(\frac{y_{1}}{f_{1}^{\prime}}\right)^{3}\left(f_{1}^{\prime}-d_{1}\right) \alpha \\
C_{0,0, \mathrm{af}}= & \frac{1}{16}\left(\frac{y_{1}}{f_{1}^{\prime}}\right)^{2} \frac{d_{1}^{2}}{f_{1}^{\prime}-d_{1}}\left(1+b_{\mathrm{s} 2}\right) \\
C_{1,0, \mathrm{af}}= & \frac{1}{4}\left(\frac{y_{1}}{f_{1}^{\prime}}\right)^{2}\left[\alpha\left(2 f_{1}^{\prime}-d_{1}\right)\right. \\
& \left.+\delta\left(1+\frac{d_{1}}{2\left(f_{1}^{\prime}-d_{1}\right)}\left(1+b_{\mathrm{s} 2}\right)\right)\right] \\
C_{2,0, \mathrm{af}}= & \frac{1}{4}\left(\frac{y_{1}}{f_{1}^{\prime}}\right)^{2}\left[\alpha^{2}\left(f_{1}^{\prime}-d_{1}\right)+\alpha \delta\right. \\
& \left.+\delta^{2} \frac{1+b_{\mathrm{s} 2}}{4\left(f_{1}^{\prime}-d_{1}\right)}\right]
\end{aligned}
$$

Equations (37), (38) and (39) for focal telescopes are then also valid for afocal telescopes if all expressions in these formulae are replaced by the corresponding expressions (40) to (46) for afocal telescopes.

\subsection{Explicit expressions for astigmatism in a coma-free schiefspiegler}

\subsubsection{Telescope in focal form}

A further simplification is possible if the telescope is corrected for coma at the center of the field, that is for $u_{\text {pr1 }}=0$. The axes of M1 and M2 must then intersect at the coma-free point $P_{\mathrm{cfp}}$. The distance from the vertex of M2 to $P_{\text {cfp }}$ is denoted by $z$. The lateral decenter $\delta$ and the misalignment angle $\alpha$ are then related by $\delta=-\alpha z$. $z$ can be calculated from the requirement that the contributions to decentering coma from a pure lateral decenter $\delta$ and the simultaneous rotation $\alpha=-\delta / z$ cancel.

$c_{\text {coma }, \delta}+c_{\text {coma }, \alpha}=0$.

This gives, using the Eqs. (31) and (32),

$$
\begin{aligned}
z= & 2 L \frac{m_{2}-1}{m_{2}+1} \cdot \\
& \cdot \frac{1}{m_{2}-1-\left(m_{2}+1\right) b_{\mathrm{s} 2}-\frac{s_{\mathrm{pr} 1}}{f^{\prime}} \frac{4}{\left(m_{2}+1\right)^{2} d_{1}^{2}}\left(-f^{\prime} \zeta+L \xi\right)} .
\end{aligned}
$$

This equation shows that the position of the coma-free point depends on the stop position. If the stop is at the primary mirror or if the telescope is corrected for spherical aberration, $z$ depends, for a given telescope geometry $L$ and $m_{2}$, only on the aspheric constant $b_{\mathrm{s} 2}$ of the secondary mirror.

$$
z=2 L \frac{m_{2}-1}{m_{2}+1} \frac{1}{m_{2}-1-\left(m_{2}+1\right) b_{\mathrm{s} 2}} .
$$

If the stop is at the secondary mirror $z$ depends, for a given telescope geometry $L$ and $m_{2}$, only on the asphericity $b_{\mathrm{s} 1}$ of the primary mirror.

$$
z=2 L \frac{m_{2}^{2}-1}{2 m_{2}\left(m_{2}^{2}-1\right)-\frac{f^{\prime}}{L} m_{2}^{3}\left(1+b_{\mathrm{s} 1}\right)} .
$$

The coefficient $c_{\text {ast }}$ of third order astigmatism can then be expressed as

$c_{\mathrm{ast}}=B_{0} u_{\mathrm{pr} 1}^{2}+B_{1} u_{\mathrm{pr} 1} \alpha+B_{2} \alpha^{2}$

with

$$
\begin{aligned}
& B_{0}= C_{0} \\
& B_{1}=-\frac{1}{4}\left(\frac{y_{1}}{f^{\prime}}\right)^{2} \frac{z}{d_{1}}\left[\frac{f^{\prime} d_{1}}{L} \frac{\left(m_{2}+1\right)^{3}}{m_{2}-1} b_{\mathrm{s} 2}\right. \\
&+\frac{s_{\mathrm{pr} 1}}{f^{\prime}}\left(f^{\prime}+2 d_{1} \xi\right. \\
&\left.\left.+2 \frac{2 L+\left(m_{2}+1\right) d_{1}}{\left(m_{2}-1\right) L}(-f \zeta+L \xi)\right)\right] \\
& B_{2}= \frac{1}{4}\left(\frac{y_{1}}{f^{\prime}}\right)^{2}\left(\frac{z}{d_{1}}\right)^{2}\left[\frac{d_{1}^{2}}{L}\left(\frac{m_{2}+1}{m_{2}-1}\right)^{2} b_{\mathrm{s} 2} \xi\right. \\
&+\frac{s_{\mathrm{pr} 1}}{f^{\prime}} \frac{2 d_{1}}{L}\left(\frac{m_{2}+1}{m_{2}-1}\right)^{2} b_{\mathrm{s} 2}(-f \zeta+L \xi) \\
&+\left(\frac{s_{\mathrm{pr} 1}}{f^{\prime}}\right)^{2}(-f \zeta+L \xi) \\
&\left.\left(\frac{4}{\left(m_{2}-1\right)^{2}\left(m_{2}+1\right)} \frac{-f \zeta+L \xi}{L}-1\right)\right] .
\end{aligned}
$$

Equations (52), (53) and (54) show that the parameters $B_{i}$ are proportional to $\left(z / d_{1}\right)^{i}$. At a first glance they seem to be linear or quadratic equations in $s_{\mathrm{pr} 1} / f^{\prime}$. This is only the case for $B_{0}$ since the distance $z$ of the coma-free point appearing in $B_{1}$ and $B_{2}$ depends itself on the stop position, as can be seen from Eq. (49).

By introducing $z=-\delta / \alpha$ in the Eqs. (52), (53) and (54) the coefficient of third order astigmatism can be expressed as a polynomial in $u_{\mathrm{pr} 1}$ and $\delta$, that is

$c_{\mathrm{ast}}=B_{0} u_{\mathrm{pr} 1}^{2}+B_{1}^{(\delta)} u_{\mathrm{pr} 1} \delta+B_{2}^{(\delta)} \delta^{2}$.

It is easy to see that now the parameter $B_{1}^{(\delta)}$ is a linear function and the parameters $B_{0}$ and $B_{2}^{(\delta)}$ are quadratic functions in $s_{\text {pr1 }}$.

Exactly as with $C_{0}, C_{1}$ and $C_{2}$ the parameters $B_{0}, B_{1}$ and $B_{2}$ depend only on the asphericity $b_{\mathrm{s} 2}$ of $\mathrm{M} 2$, if the stop is at the primary mirror, and only on the asphericity $b_{\mathrm{s} 1}$ of $\mathrm{M} 1$, if the stop is at the secondary mirror.

Since the coupling between $\alpha$ and $\delta$ through $z$ involves the expression for spherical aberration, the symmetry of the stop-shift terms has disappeared. But, the telescope will usually be corrected for spherical aberration. In this case all terms containing $-f^{\prime} \zeta+L \xi$ vanish. Then, with the additional definitions

$B_{0,0}=\frac{1}{4}\left(\frac{y_{1}}{f^{\prime}}\right)^{2}\left[\frac{f^{\prime}}{L}\left(f^{\prime}+d_{1}\right)+\frac{d_{1}^{2}}{L} \xi\right]$ 
$B_{1,0}=-\frac{1}{4}\left(\frac{y_{1}}{f^{\prime}}\right)^{2} \frac{f^{\prime} d_{1}}{L} \frac{\left(m_{2}+1\right)^{3}}{m_{2}-1} b_{\mathrm{s} 2}$

$B_{2,0}=\frac{1}{4}\left(\frac{y_{1}}{f^{\prime}}\right)^{2} \frac{d_{1}^{2}}{L}\left(\frac{m_{2}+1}{m_{2}-1}\right)^{2} b_{\mathrm{s} 2} \xi$.

Equations (52), (53) and (54) reduce to

$B_{0}=B_{0,0}-\frac{s_{\text {pr1 }}}{y_{1}} c_{\text {coma,cen }}^{*}$

$B_{1}=\frac{z}{d_{1}}\left(B_{1,0}+\frac{s_{\mathrm{pr} 1}}{y_{1}} c_{\text {coma,cen }}^{*}\right)$

$B_{2}=\left(\frac{z}{d_{1}}\right)^{2} B_{2,0}$.

The distance $z$ from the secondary mirror to the comafree point and the expression $c_{\text {coma,cen }}^{*}$ are now no longer dependent on the stop position. Therefore, $B_{0}$ and $B_{1}$ are linear functions of the stop position and $B_{2}$ is independent of the stop position.

\subsubsection{Telescope in afocal form}

The Eqs. (59), (60) and (61), all valid for focal telescopes corrected for spherical aberration, can be converted into equations valid for afocal telescopes, also corrected for spherical aberration, by using Eqs. (21) and (19) and letting $m_{2}$ go to infinity. The distance from M2 to the comafree point is then

$z=2 \frac{f_{1}^{\prime}-d_{1}}{1-b_{\mathrm{s} 2}}$

For a classical or aplanatic afocal telescope the coma-free point is in the focus of M1. The astigmatism parameters are given by

$$
\begin{aligned}
B_{0}= & \frac{1}{4}\left(\frac{y_{1}}{f_{1}^{\prime}}\right)^{2}\left(1+b_{\mathrm{s} 2}\right)\left[\frac{1}{4} \frac{d_{1}^{2}}{f_{1}^{\prime}-d_{1}}+s_{\mathrm{pr} 1} \frac{d_{1}}{2 f_{1}^{\prime}}\right] \\
B_{1}= & -\frac{1}{4}\left(\frac{y_{1}}{f_{1}^{\prime}}\right)^{2} \frac{1}{1-b_{\mathrm{s} 2}}\left[2 f_{1}^{\prime} b_{\mathrm{s} 2}\right. \\
& \left.+s_{\mathrm{pr} 1} \frac{f_{1}^{\prime}-d_{1}}{f_{1}^{\prime}}\left(1+b_{\mathrm{s} 2}\right)\right] \\
B_{2}= & \frac{1}{4}\left(\frac{y_{1}}{f_{1}^{\prime}}\right)^{2} \frac{1+b_{\mathrm{s} 2}}{\left(1-b_{\mathrm{s} 2}\right)^{2}} b_{\mathrm{s} 2}\left(f_{1}^{\prime}-d_{1}\right) .
\end{aligned}
$$

For a Mersenne telescope with $b_{\mathrm{s} 1}=b_{\mathrm{s} 2}=-1$ one obtains immediately $B_{0}=B_{2}=0$. This gives therefore pure linear astigmatism which is proportional to the misalignment angle $\alpha$ with the center of the pattern at the center of the field. This is a nice example of the general statement by Shack \& Thompson (1980) that a system which is free of astigmatism in the centered configuration will show either linear or constant astigmatism in the decentered configuration.

\subsection{Conclusions for specific types of telescopes and stop positions}

\subsection{Specific stop positions at coma-free schiefspieglers}

For a general coma-free schiefspiegler the following conclusions can be drawn for the stop positions at the primary and secondary mirrors.

\section{- Stop at the primary mirror}

For a spherical secondary $\left(b_{\mathrm{s} 2}=0\right)$ the Eqs. (53) and (54) show that one has, as for a centered system, $B_{1}=B_{2}=0$. Because of $z=2 f_{2}^{\prime}$ the coma-free point is at the center of curvature of M2. Since a rotation of a spherical mirror around its center of curvature does not change the optical characteristics of the telescope, the astigmatism pattern has to be the same as the one of the centered system.

If $\xi=0$, one has $B_{2}=0$, that is one node is at the position of the image corresponding to the field center.

- Stop at the secondary mirror

If one uses the substitution $s_{\mathrm{pr} 1} / f=-d_{1} / L$ in Eq. (54) one can show that $B_{2}$ is proportional to $\zeta$. Therefore, for a coma-free schiefspiegler with a parabolic primary mirror one node is at the position of the image corresponding to the field center.

\subsection{Specific types of coma-free schiefspieglers corrected for spherical aberration}

From the general formulae given above more specific conclusions can be drawn if the telescopes is corrected for spherical aberration. In all cases we will also discuss the results in the limit of large magnifications, defined here as $m_{2} \rightarrow \infty$ together with a finite distance $L$ from the secondary mirror to the focus. The semi-diameter $y_{2}$ of M2, its radius of curvature $2 f_{2}^{\prime}$ and the difference between $f_{1}^{\prime}$ and $d_{1}$ will go to zero. This is different from the case of an afocal telescope, where $L$ goes to infinity, while $y_{2}, 2 f_{2}^{\prime}$

\begin{tabular}{|c|c|c|}
\hline & Stop at M1 & Stop at M2 \\
\hline$B_{0} \rightarrow$ & $+\frac{1}{1} \frac{y_{1}^{2}}{T}\left(1+b_{\mathrm{s} 2}\right) m_{2}$ & $-\frac{1}{1} \frac{y_{1}^{2}}{T}\left(1+b_{\mathrm{s} 2}\right) m_{2}$ \\
\hline & $\begin{array}{c}16 L \\
+\frac{1}{4} \frac{y_{1}^{2}}{L}\end{array}$ & $\begin{array}{c}16 L \\
+\frac{1}{4} \frac{y_{1}^{2}}{L}\end{array}$ \\
\hline \multirow{2}{*}{$B_{1} \rightarrow$} & $-\frac{1}{y_{1}^{2}} \quad b_{\mathrm{s} 2}$ & $\underline{1} \underline{y}_{1}^{2}$ \\
\hline & $-\overline{2} \overline{f_{1}^{\prime}} \overline{1-b_{\mathrm{s} 2}}$ & $\overline{4} \overline{f_{1}^{\prime}}$ \\
\hline$B_{2} \rightarrow$ & 0 & 0. \\
\hline
\end{tabular}
and $f_{1}^{\prime}-d_{1}$ remain finite. For the limit case with finite $L$ we get from Eqs. (56) to (58) the following expressions for the astigmatism parameters:

We now discuss a few telescope types.

\section{- Classical telescope}

The primary mirror is parabolic and $\xi=0$. Therefore $B_{2}$ vanishes. The consequence of this is that one of 
the two nodes is exactly at the image position for an object on the axis of the primary mirror, that is with $u_{\text {pr1 }}=0$.

For the limit of large magnifications one has $b_{\mathrm{s} 2} \rightarrow-1$ and therefore from Eq. (66) independently of the stop position:

$$
\begin{aligned}
& B_{0} \rightarrow \frac{1}{4} \frac{y_{1}^{2}}{L} \\
& B_{1} \rightarrow \frac{1}{4} \frac{y_{1}^{2}}{f_{1}^{\prime}} \\
& \frac{B_{1}}{B_{0}} \rightarrow \frac{L}{f_{1}^{\prime}} .
\end{aligned}
$$

Since the distance $L$ between the vertex of M2 and the focus will generally be similar to the focal length of M1 the ratio $B_{1} / B_{0}$ will be close to -1 .

- Ritchey-Chretien telescope

All three parameters are independent of the stop position, since, in addition to spherical aberration, coma is zero as well. Since modern Ritchey-Chretien telescopes with large values of $m_{2}$ are very close to classical telescopes, the conclusions drawn for classical telescopes will be approximately valid. That is, $B_{2}$ will be much smaller than $B_{0}$ and $B_{1}$, a point also discussed by Wilson (1999, Sect. 2.2.1), and the ratio $B_{1} / B_{0}$ will be close to -1 .

- Telescope with a spherical secondary mirror (DallKirkham)

Because of $b_{\mathrm{s} 2}=0$ the parameter $B_{2}$ is zero and $B_{1}$ is only proportional to the coma of the telescope. One node is therefore at the image position corresponding to the field center with $u_{\mathrm{pr} 1}=0$. If, in addition, the stop is at the primary mirror, $B_{1}$ is also zero. In that case the two nodes coincide and one has pure quadratic astigmatism with the center on the axis of the primary mirror.

For the limit of large magnifications one has from Eq. (66):

\begin{tabular}{ccc} 
& Stop at M1 & Stop at M2 \\
\hline$B_{0} \rightarrow+\frac{1}{16} \frac{y_{1}^{2}}{L} m_{2}$ & $-\frac{1}{16} \frac{y_{1}^{2}}{L} m_{2}$ \\
$B_{1} \rightarrow \quad 0$ & $\frac{1}{4} \frac{y_{1}^{2}}{f_{1}^{\prime}}$ \\
$\frac{B_{1}}{B_{0}} \rightarrow \quad 0$ & 0 & $-4 \frac{L}{f^{\prime}}$
\end{tabular}

Whereas $B_{1}$ converges to values independent of $m_{2}, B_{0}$ is proportional to $m_{2}$. The binodal nature of the field astigmatism diminishes therefore linearly with increasing magnification of the telescope. For a certain stop position between the primary and the secondary mirror the coefficient $B_{0}$ of quadratic astigmatism vanishes. For the VLT with the Nasmyth focus the stop would be $3.9 \mathrm{~m}$ in front of the secondary mirror. The coefficient of linear astigmatism would be small, $B_{1} \approx 22 \mu \mathrm{m} / \mathrm{deg}$, but the coefficient of field coma would be very large, $c_{\text {coma,cen }}^{*} \approx 660 \mu \mathrm{m} / \mathrm{deg}$.
- Telescope with a spherical primary mirror

For large magnifications we have in the limit $m_{2} \rightarrow \infty$ $b_{\mathrm{s} 2} \rightarrow m_{2} f_{1}^{\prime} / L$ and then from Eq. (66):

\begin{tabular}{lcc} 
& Stop at M1 & Stop at M2 \\
\hline$B_{0} \rightarrow \frac{1}{16}\left(\frac{y_{1}}{L}\right)^{2} f_{1}^{\prime} m_{2}^{2}$ & $-\frac{1}{16}\left(\frac{y_{1}}{L}\right)^{2} f_{1}^{\prime} m_{2}^{2}$ \\
$B_{1} \rightarrow$ & $\frac{1}{2} \frac{y_{1}^{2}}{f_{1}^{\prime}}$ & $\frac{1}{4} \frac{y_{1}^{2}}{f_{1}^{\prime}}$ \\
$\frac{B_{1}}{B_{0}} \rightarrow \quad 8\left(\frac{L}{f^{\prime}}\right)^{2}$ & $-4\left(\frac{L}{f^{\prime}}\right)^{2}$
\end{tabular}

$B_{1}$ converges to values independent of $m_{2}$. The limit values of $B_{0}$ are by a factor $m_{2} f_{1}^{\prime} / L$ larger than the ones for a telescopes with a spherical secondary. The binodal nature of the field astigmatism diminishes therefore quadratically with increasing magnification of the telescope.

\subsection{Numerical examples for the VLT}

In this sections we give numerical examples for the Cassegrain focus of the VLT telescope. The optical parameters used for these calculations are summarized in the following table.

\begin{tabular}{|l|r||l|r|}
\hline $2 f_{1}^{\prime}$ & $-28804.832 \mathrm{~mm}$ & $2 f_{2}^{\prime}$ & $-4553.561 \mathrm{~mm}$ \\
$b_{\mathrm{s} 1}$ & -0.996962 & $b_{\mathrm{s} 2}$ & -1.66926 \\
$y_{1}$ & $4057.50 \mathrm{~mm}$ & $y_{2}$ & $556.55 \mathrm{~mm}$ \\
\hline$d_{1}$ & $-12426.946 \mathrm{~mm}$ & & \\
$z$ & $-1997.995 \mathrm{~mm}$ & & \\
$L$ & $14926.950 \mathrm{~mm}$ & & \\
$m_{2}$ & -7.556 & & \\
\hline
\end{tabular}

If the pupil was located at the primary mirror, we would have $s_{\mathrm{pr} 1}=0$. This would give the following numerical values for the parameters $B_{0}, B_{1}$ and $B_{2}$.

$$
\begin{aligned}
& B_{0}=+71.788 \mu \mathrm{m} / \mathrm{deg}^{2} \\
& B_{1}=-84.784 \mu \mathrm{m} / \mathrm{deg}^{2} \\
& B_{2}=+0.06630 \mu \mathrm{m} / \mathrm{deg}^{2} .
\end{aligned}
$$

As has been discussed before, the value of $B_{2}$ is effectively negligible compared with the values of $B_{0}$ and $B_{1}$ and the ratio $B_{1} / B_{0}$ is approximately equal to -1 .

In reality the pupil is located at the secondary mirror. Then $s_{\mathrm{pr} 2}=0$ and from Eq. (6) one gets $s_{\mathrm{pr} 1}=$ $90600.23 \mathrm{~mm}$. This gives the following numerical values for the parameters:

$$
\begin{aligned}
& B_{0}=+86.614 \mu \mathrm{m} / \mathrm{deg}^{2} \\
& B_{1}=-87.167 \mu \mathrm{m} / \mathrm{deg}^{2} \\
& B_{2}=+0.06630 \mu \mathrm{m} / \mathrm{deg}^{2} .
\end{aligned}
$$

Since the spherical aberration is zero the value of $B_{2}$ is, as can be seen from Eq. (54), identical to the one for the stop at the primary mirror. But, there is a significant difference between the values of $B_{0}$ for the two stop positions. 


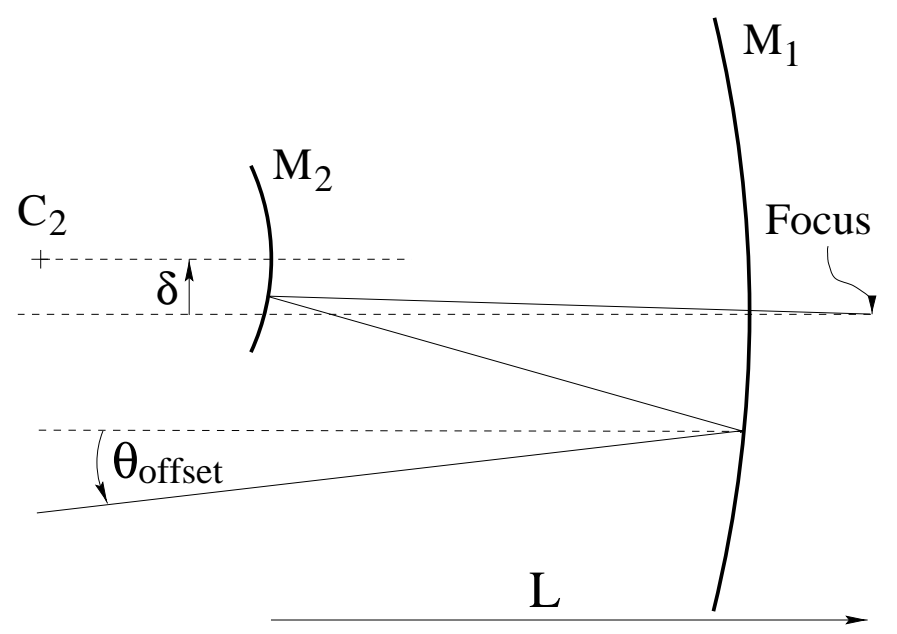

Fig. 2. Pure lateral decenter of $\mathrm{M} 2$ by $\delta . C_{2}$ is the center of curvature of M2

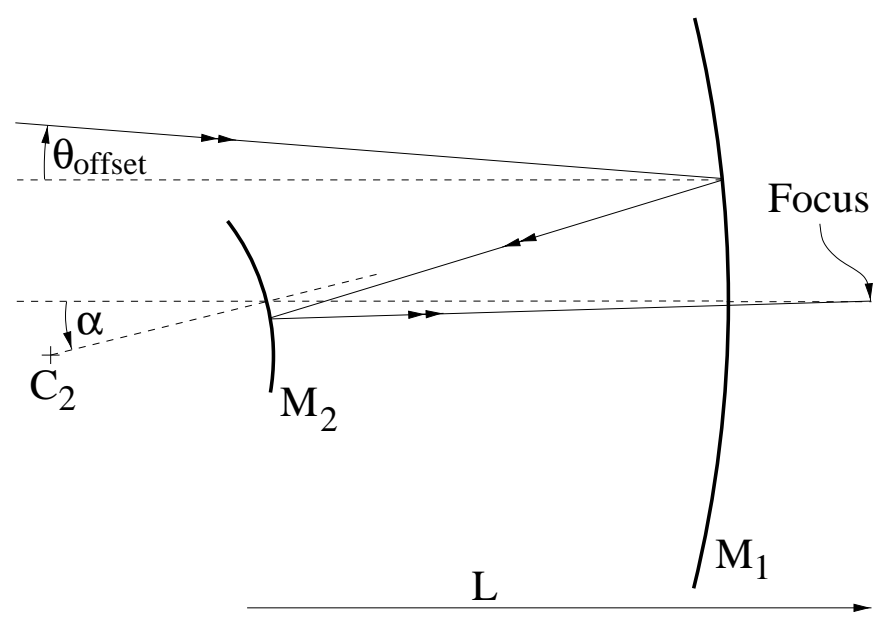

Fig. 3. Pure rotation of M2 by $\alpha$ around its vertex. $C_{2}$ is the center of curvature of M2

\section{Recalculation of the coefficients $B_{0}, B_{1}$ and $B_{2}$ for the center of the adapter}

\subsection{Introduction}

In the formulae given above the center of the field is defined by $u_{\mathrm{pr} 1}=0$ and corresponds to the direction of a star located on the M1 axis. In a decentered telescope the rays coming from such a star do not focus in the center of the adapter.

The center of the adapter is however always defined as being the center of the field in a telescope and field measurements are made with reference to this point. In this section we first calculate the offset angles of the telescope corresponding to the misalignment of M2 and then the effect of these offsets on the parameters $B_{0}, B_{1}$ and $B_{2}$.
3.2. Depointing of the telescope for a pure lateral decentering of $\mathrm{M}^{2}$

- Depointing for a pure lateral decentering of M2 If M2 is laterally decentered by $\delta$ (see Fig. 2) the star centered in the adapter is producing an angle $\theta_{\text {offset,d }}$ with the M1 axis:

$\theta_{\text {offset }, \mathrm{d}}=-\frac{L}{f_{2}^{\prime} f^{\prime}} \delta$.

For the VLT at the Cassegrain focus the factor in front of $\delta$ is $-6.02510^{-5} / \mathrm{mm}$.

- Depointing for M2 tilted around its vertex

If M2 is tilted by an angle $\alpha$ around its pole (see Fig. 3), the star centered on the adapter is producing an angle $\theta_{\text {offset,t }}$ with the M1 axis.

$\theta_{\text {offset }, \mathrm{t}}=\frac{-2 L}{f} \alpha$.

For the VLT at the Cassegrain focus the factor in front of $\alpha$ is -0.2743 .

- Depointing for M2 rotated around the coma-free point When M2 is rotated around its coma-free point by an angle $\alpha$ we have a combination of pure tilt and lateral decentering with $\delta=-\alpha z$. The resulting offset of the telescope is

$$
\begin{aligned}
\theta_{\text {offset }, \mathrm{cfp}} & =\theta_{\text {offset }, \mathrm{t}}+\theta_{\text {offset }, \mathrm{d}} \\
& =T \alpha
\end{aligned}
$$

with

$T=\frac{-L}{f}\left(2-\frac{z}{f_{2}^{\prime}}\right)$.

For the VLT at the Cassegrain focus one has $T=$ -0.1539 .

3.3. New coefficients $B_{0}, B_{1}$ and $B_{2}$ with reference to the center of the adapter

To get the coefficients $B_{0}, B_{1}$ and $B_{2}$ with the reference of the field in the center of the adapter, we define new field angles as

$\theta^{\prime}=\theta-T \alpha$.

Replacing then $\theta$ by $\theta^{\prime}+T \alpha$ in the Eqs. (1) and (2) leads to

$$
\begin{aligned}
Z_{4}^{\mathrm{sys}}= & B_{0}^{\prime}\left(\theta_{x}^{\prime 2}-\theta_{y}^{\prime 2}\right)+B_{1}^{\prime}\left(\theta_{x}^{\prime} \alpha_{x}-\theta_{y}^{\prime} \alpha_{y}\right) \\
& +B_{2}^{\prime}\left(\alpha_{x}^{2}-\alpha_{y}^{2}\right) \\
Z_{5}^{\mathrm{sys}}= & 2 B_{0}^{\prime} \theta_{x}^{\prime} \theta_{y}^{\prime}+B_{1}^{\prime}\left(\theta_{x}^{\prime} \alpha_{y}+\theta_{y}^{\prime} \alpha_{x}\right)+2 B_{2}^{\prime} \alpha_{x} \alpha_{y} .
\end{aligned}
$$

Equations (77) and (78) are similar to Eqs. (1) and (2), but with different coefficients $B_{0}^{\prime}, B_{1}^{\prime}$ and $B_{2}^{\prime}$. The new parameters $B_{0}^{\prime}, B_{1}^{\prime}$ and $B_{2}^{\prime}$ are given by

$B_{0}^{\prime}=B_{0}$

$B_{1}^{\prime}=B_{1}+2 B_{0} T$

$B_{2}^{\prime}=B_{2}+B_{1} T+B_{0} T^{2}$. 
For the VLT with the pupil at M1

$$
\begin{array}{ll}
B_{0}=+71.788 \mu \mathrm{m} / \mathrm{deg}^{2} & B_{0}^{\prime}=+71.788 \mu \mathrm{m} / \mathrm{deg}^{2} \\
B_{1}=-84.783 \mu \mathrm{m} / \mathrm{deg}^{2} & B_{1}^{\prime}=-106.888 \mu \mathrm{m} / \mathrm{deg}^{2} \\
B_{2}=+0.0663 \mu \mathrm{m} / \mathrm{deg}^{2} & B_{2}^{\prime}=+14.821 \mu \mathrm{m} / \mathrm{deg}^{2} .
\end{array}
$$

For the VLT with the pupil at M2

$$
\begin{array}{ll}
B_{0}=+86.614 \mu \mathrm{m} / \mathrm{deg}^{2} & B_{0}^{\prime}=+86.614 \mu \mathrm{m} / \mathrm{deg}^{2} \\
B_{1}=-87.167 \mu \mathrm{m} / \mathrm{deg}^{2} & B_{1}^{\prime}=-113.837 \mu \mathrm{m} / \mathrm{deg}^{2} \\
B_{2}=+0.0663 \mu \mathrm{m} / \mathrm{deg}^{2} & B_{2}^{\prime}=+15.539 \mu \mathrm{m} / \mathrm{deg}^{2}
\end{array}
$$

In both cases the new coefficients $B_{1}^{\prime}$ are approximately $30 \%$ larger than the original coefficients $B_{1}$.

\subsection{Effects of the correction of coma at the center of the adapter}

The formulae in Sect. 2.3 have been derived under the assumption that the telescope was a coma-free schiefspiegler. This means that the telecope is corrected for coma for the field center with $u_{\mathrm{pr} 1}=0$. But, in reality the coma correction will ensure that the telescope is free of coma at the center of the adapter. The formulae derived above can therefore, strictly speaking, not be applied to non-aplanatic telescopes. But, a short calculation will show that, in practice, the inaccuracies introduced by this effect are negligible.

Equation (74) gives the relationship between the misalignment angle and the corresponding field angle. The coefficient of coma for this field angle can then be calculated from Eq. (30). Finally, one needs the coefficient $c_{\mathrm{coma}, \mathrm{coc}}$ of coma generated by a rotation around the center of curvature of M2 by an angle $\varphi$. This can be derived by combining the expressions for coefficients of coma generated by a pure lateral decentering by $\delta$ and a rotation around the vertex of M2 by an angle $u_{\mathrm{pr} 2}$ (see Eqs. (31) and (32)). If $\delta$ and $u_{\mathrm{pr} 2}$ are related by $\delta=-2 f_{2}^{\prime} u_{\mathrm{pr} 2}$ the total effect is a rotation around the center of curvature of M2. One then obtains

$c_{\text {coma }, \mathrm{coc}}=\frac{1}{4}\left(\frac{y_{1}}{f^{\prime}}\right)^{3}\left(m_{2}-1\right) \frac{f^{\prime} L}{z} T \varphi$.

Combining the three Eqs. (74), (30) and (80) gives the rotation angle $\varphi$ of M2 which shifts the coma correction from the field center with $u_{\mathrm{pr} 1}=0$ to the center of the adapter.

$$
\begin{aligned}
\varphi & =-\frac{\left(2 d_{1} \xi+f^{\prime}\right) z}{\left(m_{2}-1\right) f^{\prime} L} \alpha \\
& =-\frac{2 \alpha}{\left(m_{2}+1\right)\left[m_{2}-1-\left(m_{2}+1\right) b_{\mathrm{s} 2}\right]} \frac{2 d_{1} \xi+f^{\prime}}{f^{\prime}} .
\end{aligned}
$$

For a classical telescope this reduces to the simple expression

$\varphi_{\text {class }}=-\frac{1}{m_{2}\left(m_{2}-1\right)} \alpha$.
Since the VLT is optically very close to a classical telescope and $m_{2}=-7.556, \varphi$ is approximately fifty times smaller than $\alpha$. This shows that the difference between a schiefspiegler with coma corrected for the center of the field and one with coma corrected for the center of the adapter is negligible. The formulae for field astigmatism derived above for a schiefspiegler free of coma at the center of the field can therefore also be used for a schiefspiegler with coma corrected for the center of the adapter.

\subsection{Simulation for the ESO 3.6 m telescope}

As an example we checked our formula with a simulation done with Zemax for the $3.6 \mathrm{~m}$ telescope on La Silla. We simulated M2 tilted around the coma-free point by an angle $\alpha=0.21136^{\circ}$, which corresponds to an unusually large decenter of $\delta=10 \mathrm{~mm}$.

The values of astigmatism calculated at different field positions by Zemax were entered into our fitting software. Two fittings were done, one with the set of parameters $B_{0}, B_{1}, B_{2}$ for the original field center and one with the set $B_{0}^{\prime}, B_{1}^{\prime}$ and $B_{2}^{\prime}$ for the field center at the center of the adapter. With the first set we find $\alpha=0.3867^{\circ}$ with a residual wavefront rms of $697.2 \mathrm{~nm}$. With the second set we find $\alpha=0.21041^{\circ}$, very close to the input value, with a small residual $\mathrm{rms}$ of $10.2 \mathrm{~nm}$.

\section{Misalignment measurements at the VLT}

\subsection{General procedure}

The VLT has both Nasmyth and Cassegrain focii. It is optimised to be a Ritchey-Chretien telescope at the Nasmyth focus. The Cassegrain focus has a different aperture ratio from that of the Nasmyth focus. To switch from the Nasmyth to the Cassegrain focus one has to change the distance between the mirrors (done by refocussing with M2) and bend the primary mirror to remove spherical aberration. In its Cassegrain configuration the telescope is no longer aplanatic. Furthermore, the stop is at the secondary mirror. For the measurement of the misalignment using the features of the field astigmatism one therefore has to use the constants $B_{0}^{\prime}, B_{1}^{\prime}$ and $B_{2}^{\prime}$ given at the end of Sect. 3.3.

The measurement procedure is then as follows. The telescope is first corrected for coma at the center of the adapter. With $B_{0}^{\prime}, B_{1}^{\prime}$ and $B_{2}^{\prime}$ known, the values for $\alpha_{x}$ and $\alpha_{y}$ could, in principle, be obtained from one measurement of $Z_{4}^{\text {sys }}$ and $Z_{5}^{\text {sys }}$ somewhere in the field of the telescope. But, in large telescopes field independent astigmatism can easily be generated elastically. In addition, the measurements are, for example due to local air effects, not free of noise. Therefore, it will be necessary and more accurate to do measurements at several locations in the field and obtain the values for $\alpha_{x}$ and $\alpha_{y}$ with a least squares fit. 


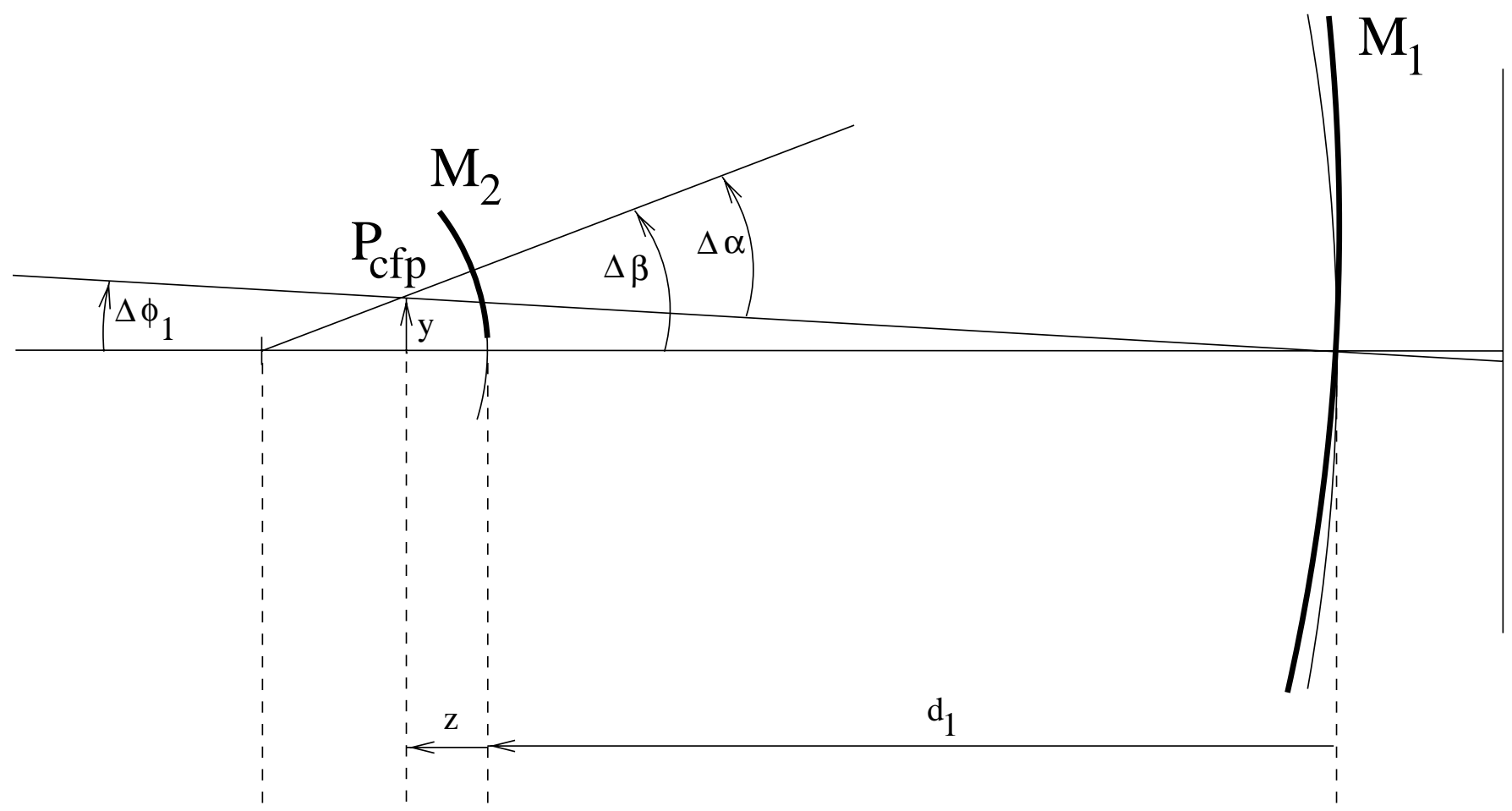

Fig. 4. Change of $\alpha$ due to a rotation of M1 around its vertex

We use typically eight measurements at evenly distributed points at the edge of the field. Such eight measurements would at least take fifteen minutes. During this time the VLT optics changes, because of elastic deformations due to changes of the zenith distance, significantly. In particular, the aberrations decentering coma and astigmatism are strongly affected. Therefore, we had to do a closed loop active optics correction at the center before each measurement at the edge and, in addition, had to subtract the variation generated by the change of altitude between the correction at the center and the measurement at the edge.

\subsection{Measurement of the accuracy of the method}

By changing the misalignment in a well defined way it is possible to estimate the accuracy of this method. The VLT has got the useful feature that the primary mirror can be moved by motors in five degrees of freedom. The only full body movement which cannot be remotely controlled, is a movement in the direction which is perpendicular to the optical and to the altitude axis. This allows that, in particular, the primary mirror can be tilted arbitrarily around its vertex. We can therefore modify the misalignment angle $\Delta \alpha$ between the primary and secondary mirror very accurately. By measuring afterwards $\Delta \alpha$ we can therefore check the accuracy of the method described above and, in addition, the validity of the theoretical parameters $B_{0}^{\prime}, B_{1}^{\prime}$ and $B_{2}^{\prime}$.

The expected change $\Delta \alpha$ of the angle between the axes of the primary and secondary mirrors due to a rotation of the primary mirror around its vertex can be deduced from Fig. 4. In an initially perfectly aligned telescope first the primary mirror has been rotated around its vertex by $\Delta \phi_{1}$. Afterwards decentering coma has been corrected by rotating the secondary mirror by $\Delta \beta$ around its center of curvature. The axes of the primary and secondary mirrors then intersect at the coma-free point $P_{\text {cfp }}$. For small angles we get

$\Delta \beta=-\frac{d_{1}-z}{2 f_{2}^{\prime}+z} \Delta \phi_{1}$.

The angle between the axes of the primary and secondary mirrors is then

$\Delta \alpha=\Delta \beta-\Delta \phi_{1}$.

The derivation of the change of the angle $\Delta \alpha$ between the axes of the primary and secondary mirrors does, at least for small initial misalignments, not depend on the actual initial state of the telescope. Equation (84) is therefore always correct.

With the VLT parameters one gets

$\Delta \alpha=-6.64 \Delta \phi_{1}$.

\subsection{Measurement data}

The first mapping was done for the initial setup of the telescope. The second and third mappings were done after tilting the primary mirror around it vertex around two orthogonal axes A and B each time by $20^{\prime \prime}$.

For a rotation of the primary mirror around its vertex by $20^{\prime \prime}$ one expects a change $\Delta \alpha=132.8^{\prime \prime}$. 
In addition, a rotation around the vertex will shift the whole pattern due to the tilt of the M1 axis, but the shift is only of the order of $0.5^{\prime \prime}$ on the sky and therefore negligible.

The three mappings gave the following results for the $x$ - and $y$-components of $\alpha$ (all figures in arcseconds).

\begin{tabular}{|l||r|r||r|r||r|}
\hline$\Delta \phi$ & $\alpha_{x}$ & $\alpha_{y}$ & $\Delta \alpha_{x}$ & $\Delta \alpha_{y}$ & $\Delta \alpha$ \\
\hline 0 & 39.24 & 149.18 & & & \\
20 A & 38.52 & 285.23 & -0.72 & 136.05 & 136.05 \\
$20 \mathrm{~B}$ & 179.17 & 142.67 & 139.93 & -6.51 & 140.08 \\
\hline
\end{tabular}

The total change $\Delta \alpha$ of the misalignment is defined by $\Delta \alpha=\sqrt{\left(\Delta \alpha_{x}\right)^{2}+\left(\Delta \alpha_{y}\right)^{2}}$. The average of the measured changes of the misalignment angles $\Delta \alpha$ between the first and the second configuration on the one hand and the first and the third configuration on the other hand is $\Delta \alpha=138.07^{\prime \prime}$. The difference to the expected value $\Delta \alpha=6.64 \cdot 20^{\prime \prime}=132.8^{\prime \prime}$ is only $5.3^{\prime \prime}$. If, after a rotation of the secondary mirror around the coma free point a similar accuracy is achieved, the two nodes of the binodal field will only be $7^{\prime \prime}$ apart. At the edge of the field of $0.25^{\circ}$ this would lead to an error in the coefficient of third order astigmatism of $56 \mathrm{~nm}$, which is negligible.

\section{Summary}

Explicit expressions in terms of fundamental telescope parameters of a two mirror telescope can be derived for the coefficients describing the field dependence of third order astigmatism. Of particular importance is the dependence of the coefficients on the position of the stop. In addition, these parameters are recalculated for a reference system where the center of the adapter is at the origin. The numerical values are used to show that from the measurement of the field dependence of third order astigmatism at eight locations in the pupil the axes of the primary and secondary mirrors of the VLT can be aligned with an accuracy of approximately $7^{\prime \prime}$.

Acknowledgements. The authors would like to thank Ray Wilson for helpful comments and discussions.

\section{References}

McLeod B.A., 1996, PASP 108, 217

Schroeder D.J., 1987, Astronomical Optics. Academic, San Diego

Shack R.V., Thompson K., 1980, SPIE, Vol. 251, Optical Alignment, p. 146

Wilson R.N., 1996, Reflecting Telescope Optics I. Springer-Verlag, Berlin

Wilson R.N., 1999, Reflecting Telescope Optics II. Springer-Verlag, Berlin 